\title{
EDUCATIONAL RESEARCH IN ACTION
}

\section{Observed improvements in an intern's ability to initiate critical emergency skills in different cardiac arrest scenarios using high-fidelity simulation}

David J. Starmer, DC, Sean A. Duquette, BA, Dominic Guiliano, DC, Anthony Tibbles, DC, Andrew Miners, DC, Kevin Finn, DC, and Brynne E. Stainsby, DC

\begin{abstract}
Objective: The objective of this study was to report observed changes in an intern's ability to initiate critical emergency skills in different cardiac arrest scenarios with high-fidelity simulation over a 10-month period.

Methods: One intern's performance was retrospectively analyzed using video recordings of 4 simulations at different stages in the training program. The key outcome was the duration of time expired for 4 critical skills, including activating the emergency response system, initiating cardiopulmonary resuscitation (CPR), using an automated external defibrillator (AED), and passively administrating oxygen.

Results: The intern became more efficient in each subsequent simulation for activating the emergency response system and initiating CPR. The time to use the AED stayed relatively constant. The administration of oxygen was inconsistent. Conclusion: An improvement in the speed of applying emergency critical skills was observed with this intern. These improvements in skill may improve patient outcomes and survival rates. We propose further educational research with high-fidelity simulation in the area of assessing emergency skills.
\end{abstract}

Key Indexing Terms: Patient Simulation; Manikins; Emergency Treatment; Chiropractic; Education

J Chiropr Educ 2014;28(2):164-167 DOI 10.7899/JCE-13-33

\section{INTRODUCTION}

High-fidelity simulation (HFS) is used to help prepare students in many disciplines. ${ }^{1-5}$ HFS in this article is defined as the use of technology manikins to create a lifelike situation for which the student has total suspended disbelief, allowing the student to practice particular technical or communications skills in a safe environment. ${ }^{6}$ Simulation provides a safe approach to learning, allowing students the opportunity to practice and learn in an environment that approximates reality. ${ }^{7}$ Students have shown a preference for full-scale simulations because they can address their underlying fear, lack of confidence, and lack of exposure to clinical situations. ${ }^{8}$ While students' skills and marks have increased after a simulation experience, there have been mixed results when used in objective structured clinical examinations. ${ }^{3}$

To our knowledge, our college is one of the first in the chiropractic profession to explore the use of HFS in emergency skills training, in this case cardiopulmonary resuscitation (CPR). Earlier research at our institution reported high levels of usability, satisfaction, and achievement of outlined learning objectives with using HFS for emergency response in an environment simulating a chiropractic office. ${ }^{1}$ Our reported simulations consisted of groups of 6-9 participants, each of whom selected the simulation role (eg, doctor, spouse, receptionist, etc). While there have been many emergency simulation experiences at our institution, there has been only 1 intern who has assumed the role of doctor in an HFS more than once, and there are no others who were observed in an environment outside the simulation laboratory. This has allowed a unique opportunity to follow 1 intern's performance through 4 cardiac simulations, 3 in a simulation laboratory and 1 in a teaching clinic. Thus, the purpose of this article is to report observed changes in an intern's ability to initiate critical emergency skills in different cardiac arrest scenarios with high-fidelity simulation over a 10 -month period.

\section{METHODS}

The intern in this report completed 4 simulations during 3 different sessions. As prerequisites to enter the clinical internship and before participating in the first simulation session, the intern had been certified in basic first aid and $\mathrm{CPR}$ and completed a course in emergency care. Both 
Table 1 - Times (Seconds) to Initiate Critical Procedures, with Time 0 Being When the Manikin Became "Unconscious"

\begin{tabular}{lcccc}
\hline & Simulation 1 & Simulation 2 & Simulation 3 & Simulation 4 \\
\hline Time 9-11 activated & +3 & -43 & -58 & -63 \\
Time of first CPR compression & +114 & +17 & +32 & +38 \\
Time of first AED shock & +162 & +127 & +142 & +151 \\
Time oxygen was administered & Not completed & +72 & Not completed & +93 \\
\hline
\end{tabular}

CPR indicates cardiopulmonary resuscitation; AED, automated external defibrillator

courses involved repetitive task training, however neither used HFS.

The first session was a novel experience in the simulation laboratory and was part of the intern's academic requirements. The intern had no knowledge of events that were to take place. A high-fidelity manikin (Gaumard Susie ${ }^{\circledR}$ S2000 model, Gaumard Scientific, Miami, FL) was used to simulate a patient in cardiac arrest. This simulation allowed the intern to apply knowledge from the classroom and provide a baseline to compare all following scenarios. Following the first simulation, a debriefing took place. Feedback on the intern's performance in the role of doctor was provided, and the laboratory coordinator also demonstrated proper use of relevant equipment. During this debriefing process, a prerecorded reference video tape was also shown, offering further feedback on appropriate actions that could have occurred in the management of this type of emergency scenario. ${ }^{1}$

The second simulation occurred immediately upon completion of the debriefing process. The third session was a planned follow-up in the simulation laboratory, which occurred 8 months later. The fourth simulation session occurred outside of the simulation laboratory in one of the institution's teaching clinics 9 months after the initial simulation.

In the fourth HFS, the manikin was set up in a clinical care room, without the intern's knowledge, to observe if the skills learned in the first 3 simulations were transferable to an environment external to the simulation laboratory setting. The intern entered the room expecting to take a history and perform a physical examination on a new real patient; however, the high-fidelity manikin was in the room. The manikin reported signs and symptoms of cardiac arrest in progress while the intern conducted a history.

For this report we retrospectively analyzed the 4 recorded video sessions of the simulated cardiac arrest scenario. The videos were stored in a bank of recorded videos. The authors analyzed the videos to objectively track any improvements in emergency care competence and/or efficiency. Competence was defined by the intern's response to carry out the following actions: initiate a call to 911, initiate CPR, administer oxygen, and utilize an automated external defibrillator (AED) machine. The efficiency of these critical skills was noted by the student's response time to initiate these actions relative to when the simulated patient became unconscious and was unresponsive. All times were recorded on a standard recording sheet (Appendix A available to download at www.journalchiroed.com).
To assess competency, the manikin was deemed to be unconscious and unresponsive when the manikin's eyes closed. This was recorded as time 0 . At this point, there were no vital signs and 0 responses from the manikin to the intern acting as the doctor. Following this point, the video was stopped and the time was recorded at each point that a critical skill was initiated. The initiation of 911 was marked as the time when the intern instructed another participant to call 911. The initiation of CPR was recorded at the moment the first chest compression occurred. Time to oxygen administration was the moment the oxygen mask was placed on the patient and the oxygen tank was turned on. Finally, time to AED use was recorded as the moment the first shock was delivered. The difference between time 0 and the time of event initiation was recorded. This study was approved by Canadian Memorial Chiropractic College research ethics board.

\section{RESULTS}

The intern was more efficient in each subsequent simulation experience (Table 1). The call to 911 was initiated sooner in each subsequent simulation. In the first simulation, 911 was activated after the manikin became unconscious; however, in the following simulations, 911 was called when signs and symptoms of cardiac distress were present. The first CPR compression occurred $85 \%$ faster in the second scenario compared to the first. CPR compression was initiated during the third and fourth scenarios sooner than in the first scenario, but not as early as the simulation immediately following the debriefing session. The time to the first AED shock decreased 22\% immediately after the debriefing, but this improvement was not maintained in the third and fourth simulations. Oxygen administration was inconsistent.

\section{DISCUSSION}

In the first simulation experience, the intern performed adequately with regard to initiating the critical steps for a cardiac event. In the second simulation experience, all critical skills were initiated sooner than in the first simulation. The debriefing process helped the student recall previous educational lessons and allowed for application in a more efficient manner. In the third simulation experience, at an 8-month follow-up, the intern performed better than the baseline level but not quite as well as the second simulation. Finally, in the clinical simulation, the fourth experience, the intern performed substantially better than the baseline levels, suggesting the ability to transfer knowledge from the 
simulation laboratory into a clinical setting. This is consistent with literature demonstrating that simulation can improve skills, which is the focus of the simulation., ${ }^{2,3}$

In each subsequent scenario, the intern initiated 911 earlier. The response changed from a reactive approach (calling emergency services after the patient fell unconscious) to a more proactive approach of placing the call upon recognition of the signs and symptoms of a cardiac arrest. Earlier recognition of cardiac arrest has shown improved outcomes by initiating the chain of survival quicker. ${ }^{9}$ Given that quicker ambulance response times increase the survival rate of a patient in cardiac arrest, calling 911 earlier can increase a patient's chances of survival. ${ }^{10}$ Specifically, survival rates for initiating 911 are $6 \%$ for a 15 -minute response time, $8 \%$ for 8 minutes, and $10 \%-11 \%$ for 5 minutes. ${ }^{10}$ It must be noted that in this study we did not record the information provided on the hypothetical 911 call, only when it occurred. The proper information can allow the emergency medical dispatcher to recognize the cardiac arrest and send the appropriate medical response. ${ }^{11}$ Future research should also look into the information given during a simulated 911 call, as it may have an effect on simulation task times and may be a limitation of this work.

The intern initiated CPR quicker after the patient fell unconscious in the second experience as compared to the first. This may be due to the intern's abilities to recognize the symptoms of a cardiac arrest. Chest compressions started $85 \%$ faster in simulation 2 compared to simulation 1. The time to start CPR in simulations 3 and 4 , while slower than the second simulation, were $72 \%$ and $67 \%$ faster than the first simulation. In simulation 2, CPR started with the patient on the examination table, while in stimulations 3 and 4 CPR started after the patient was transferred to the ground, which may account for the slower timing in the latter 2 simulations. According to the 2010 American Heart Association guidelines for CPR, early initiation of CPR can double or triple a patient's chances of survival. ${ }^{9}$ It is possible, therefore, that the decrease in the amount of time to initiate CPR after receiving feedback, as seen in this report, may positively affect the survival rate of a cardiac arrest patient.

The timing of the first AED shock was $22 \%$ faster in the second experience, after the debriefing process, but regressed back toward baseline in the third and fourth scenarios, with only a $12 \%$ and $7 \%$ improvement, respectively. It should be noted however, that using AED within 4 minutes of cardiac arrest improves survival rates ${ }^{12}$ and that in all scenarios the intern initiated AED well within this time frame.

The administration of oxygen was the only skill that did not demonstrate a pattern of improvement or consistent application. Passive oxygen administration has been shown to increase cardiac arrest survival rates in witnessed events compared to bag-mask-valve ventilation $(38.2 \%$ to $25.8 \%) .{ }^{13}$ The student did not administer oxygen initially or during the third simulation. This may be reflective of the placement of the equipment, as the intern administered it when it was it was visible and easily accessible. Additionally, it is possible that the intern lacked training with respect to oxygen administration for cardiac arrest. Typically, first-aid and CPR certification is focused on CPR and AED administration, with minimal emphasis on passive oxygen. ${ }^{14}$ Lack of familiarity and/or decreased understanding of the importance of oxygen administration may be another reason for its inconsistent administration.

This study illustrated how HFS may be used to prepare an intern for emergency situations and how the HFS environment may provide students with a near real-life scenario to practice emergency skills and clinical decision processes without the fear of negative consequences. Literature has shown that the development of technical emergency skills increases patient outcomes and survival rates. ${ }^{9,10,13,14}$ Reporting on how this intern's efficiency improved with the use of HFS is worthy of mention and deserving of further investigation as a means of increasing patient outcomes and survival rates. Publishing this type of research will, it is hoped, promote further questions and investigations to assess HFS and its use in chiropractic education.

The focus of the study was to describe objective measurements of critical technical skills. It did not include more subjective assessments of the simulation process such as proficiency of the critical skills or maintaining control over the office. A more comprehensive way of assessing student performance is an important area of future research. By design, this study is retrospective in nature and, as such, cannot draw conclusions about the association between the improved efficiency of performance and the simulation laboratory experience. Currently, it is not known if the improvements are due to the HFS experience or the repetition of practical skills. Therefore, in the future, prospective studies with a larger sample size could add valuable information regarding the role of simulation experiences in health care education.

\section{CONCLUSION}

After an HFS exercise, followed by an educational debriefing component, a student's efficiency at initiating critical emergency care procedures increased and was maintained in subsequent planned and impromptu simulations. These improvements in skill efficiency may lead to an increased chance of survival for a patient in a cardiac event. Further research is warranted to determine if other students are at this level of competency and to what extent simulation can enhance or further develop their skills.

\section{CONFLICT OF INTEREST}

The authors have no conflicts of interest to declare.

\section{About the Authors}

All of the authors are with the Canadian Memorial Chiropractic College. Address correspondence to David Starmer, Canadian Memorial Chiropractic College, 6100 Leslie Street, Toronto, Ontario, M2H 3J1, Canada; 
dstarmer@cmcc.ca. This article was received September 30, 2013, revised December 12, 2013, and January 7, 2014, and accepted January 24, 2014.

(C) 2014 Association of Chiropractic Colleges

\section{REFERENCES}

1. McGregor M, Giuliano D. Manikin-based clinical simulation in chiropractic education. J Chiropr Educ. 2012;26(1):14-23.

2. McGregor C, Paton C, Thomson C, Chandratilake M, Scott H. Preparing medical students for clinical decision making: a pilot study exploring how students make decisions and the perceived impact of a clinical decision making teaching intervention. Med Teach. 2012;34:e508-e517.

3. Yuan HB, Williams BA, Ye QH. A systematic review of selected evidence on improving knowledge and skills through high-fidelity simulation. Nurs Educ Today. 2012;32:294-298.

4. Corbridge S, Robinson FP, Tiffen J, Corbridge T. Online learning versus simulation for teaching principles of mechanical ventilation to nurse practitioner students. Int J of Nurs Educ Scholarsh. 2010; 7: Article 12. doi: 10.2202/1548-923X.1976.

5. Wayne DB, Butter J, Siddall VJ, et al. Simulationbased training of internal medicine residents in advanced cardiac life support protocols: a randomized trial. Teach Learn Med. 2005;7:202-208.
6. Gaba, D. The future vision of simulation in health care. Qual Saf Health Care. 2004;13:2-10.

7. Gordon JA, Oriol NE, Cooper JB. Bringing good teaching cases "to life": a simulator-based medical education service. Acad Med. 2004;79(1):23-27.

8. Seropian M, Brown K, Gavilanes J, Driggers B. Simulation: not just a manikin. J Nurs Educ. 2004;43: 164-169.

9. Morrison LJ, Kierzek G, Diekema DS, et al. 2010 American Heart Association Guidelines for cardiopulmonary resuscitation and emergency cardiovascular care. Circulation. 2010;122(18 Suppl 3):S665-S675. doi: 10.1161/CIRCULATIONAHA.110.970905.

10. Pell JP, Sirel JM, Marsden AK, Ford I, Cobbe SM. Effect of reducing ambulance response times on deaths from out of hospital cardiac arrest: cohort study. BMJ 2001;322:1385-1388.

11. Castrén M, Bohm K, Kvam AM, et al. Reporting of data from out-of-hospital cardiac arrest has to involve emergency medical dispatching - taking the recommendations on reporting OHCA the Utstein style a step further. Resuscitation. 2011;82:1496-1500.

12. Weisfeldt ML, Becker LB. Resuscitation after cardiac arrest: a 3-phase time-sensitive model. JAMA 2002; 288:3035-3038.

13. Bobrow BJ, Ewy GA, Clark L, et al. Passive oxygen insufflation is superior to bag-valve-mask ventilation for witnessed ventricular fibrillation out-of-hospital cardiac arrest. Ann Emerg Med. 2009;54:656-663.

14. St. John Ambulance. First Aid Reference Guide. Toronto, ON: St. John Ambulance; 2011. 